\title{
Preface: The SINTEX Project
}

The project called «Decadal and interdecadal climate variability: Scales INTeractions EXperiments (SINTEX)» was conceived in 1997, stemming from a series of discussions and meetings within the EUROCLIVAR initiative, a concerted action of the IV Framework Program of the European Union that involved most of the major European laboratories active in the field of climate simulations. The original idea was to address the issue of how different scales of phenomena interact with each other in determining the characteristics of the Earth's climate variability. It was identified early that the interannual time scales act as a pivot, allowing strong interaction towards the longer decadal time scales and at the same time

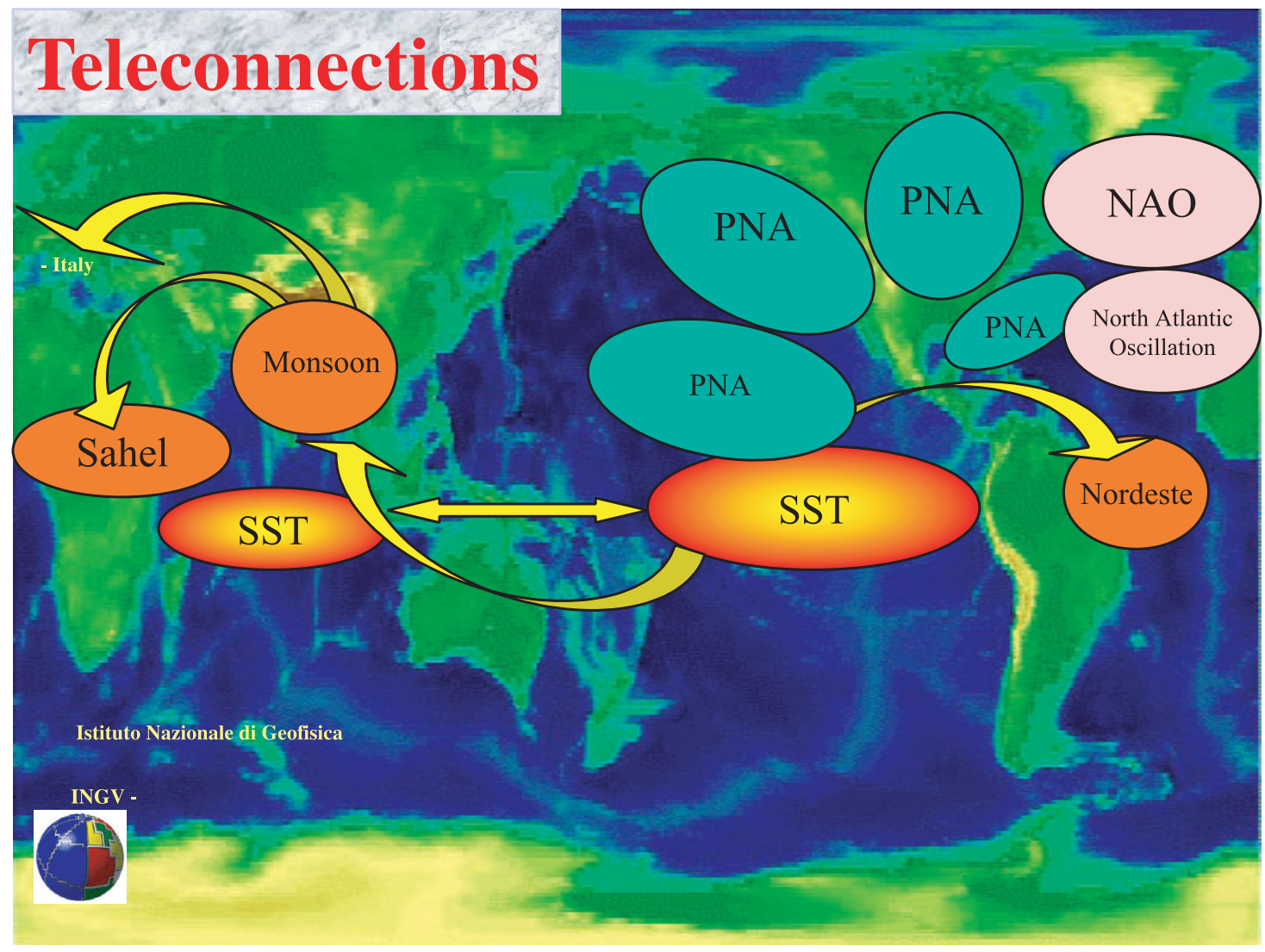

Fig. 1. The interactions between atmosphere and oceans in the tropics dominate the variability at interannual scales. Wavetrains of anomaly stem from the Pacific into the mid-latitudes, as the Pacific North American Pattern (PNA). The tropics are connected through the Pacific SST influence on the Indian Ocean SST and the monsoon, Sahel and Nordeste precipitation. The monsoon area is also connected to the Mediterranean region. Also shown is the North Atlantic Oscillation a major mode of variability in the Euro-Atlantic sector whose coupled nature is still under investigation. 
intensely interacting with the intraseasonal variability at the other end of the time spectrum. Simulating this variability is crucial to several large applications that require an accurate and timely representation of the web of interactions among time scales (fig. 1).

Seasonal forecasts are an example of how the success of the simulations is influenced by the interactions between intraseasonal variability and the slower interannual variability. Full identification of the domain of predictability that we can expect given the strongly turbulent nature of the interactions is a still matter of investigation. Climate scenarios represent another case in which the effect of rising concentrations of greenhouse gases will not result in a slow adjustment of the mean, but rather in a remodulation and modification of the space-time probability distributions of the interannual variability statistics. A realistic simulation of the interannual variability can therefore be considered a prerequisite, a prevalidation parameter, for models graduating to the simulation of future climate scenarios.

Building on the discussions during and after the EUROCLIVAR initiative, a group of investigators started to devise a more concrete plan, implementing a program of numerical

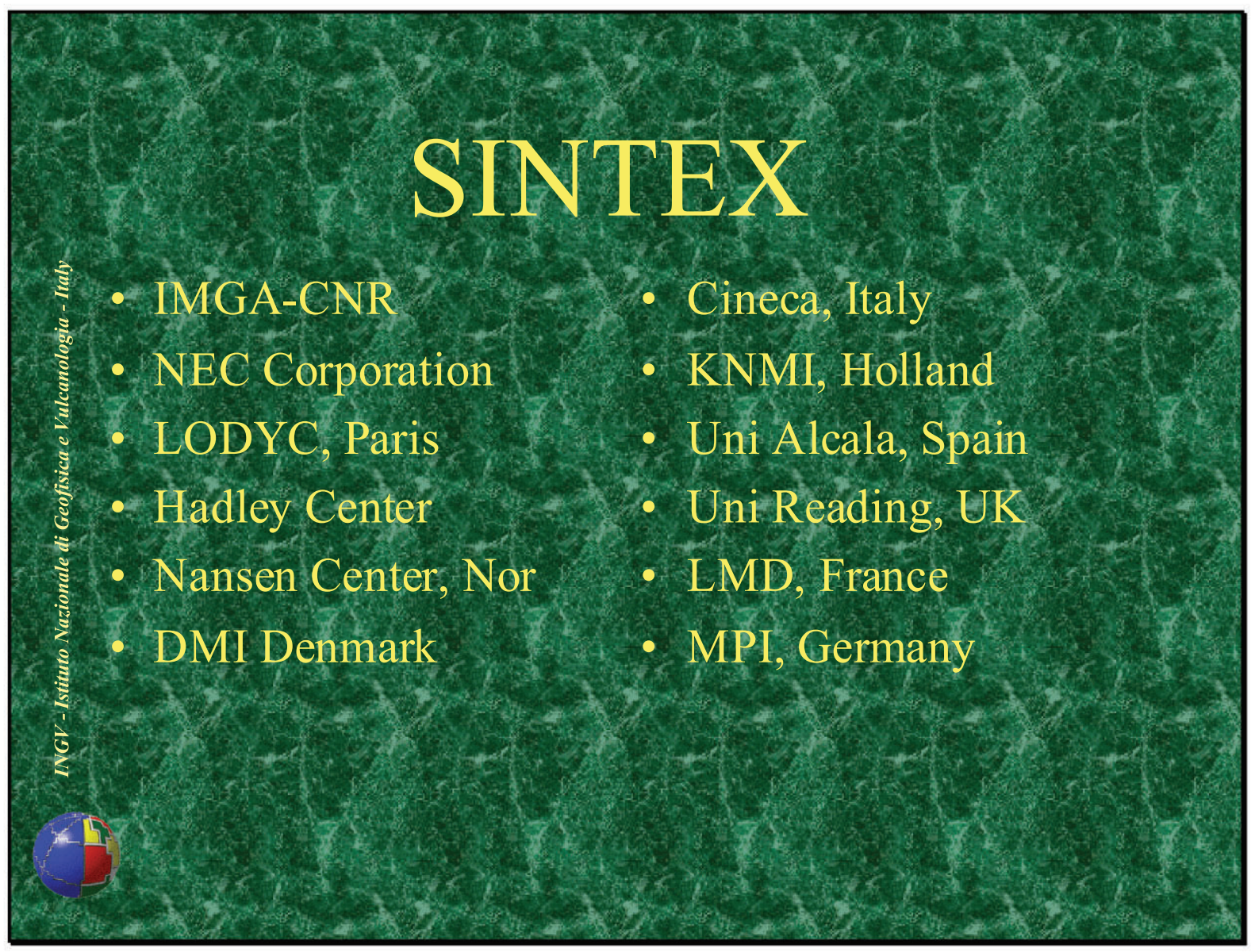

Fig. 2. The partners of SINTEX in the original slide. IMGA-CNR, Italy; NEC Supercomputing, Germany; LODYC, France; Hadley Center, U.K.; Nansen Center, Norway; Danish Meteorological Institute, Denmark; CINECA, Italy; KNMI, Holland; Universidad de Alcalá, Spain; University of Reading, U.K.; Laboratoire de Météorologie Dynamique, France; Max-Planck Institute für Meteorologie, Germany. 


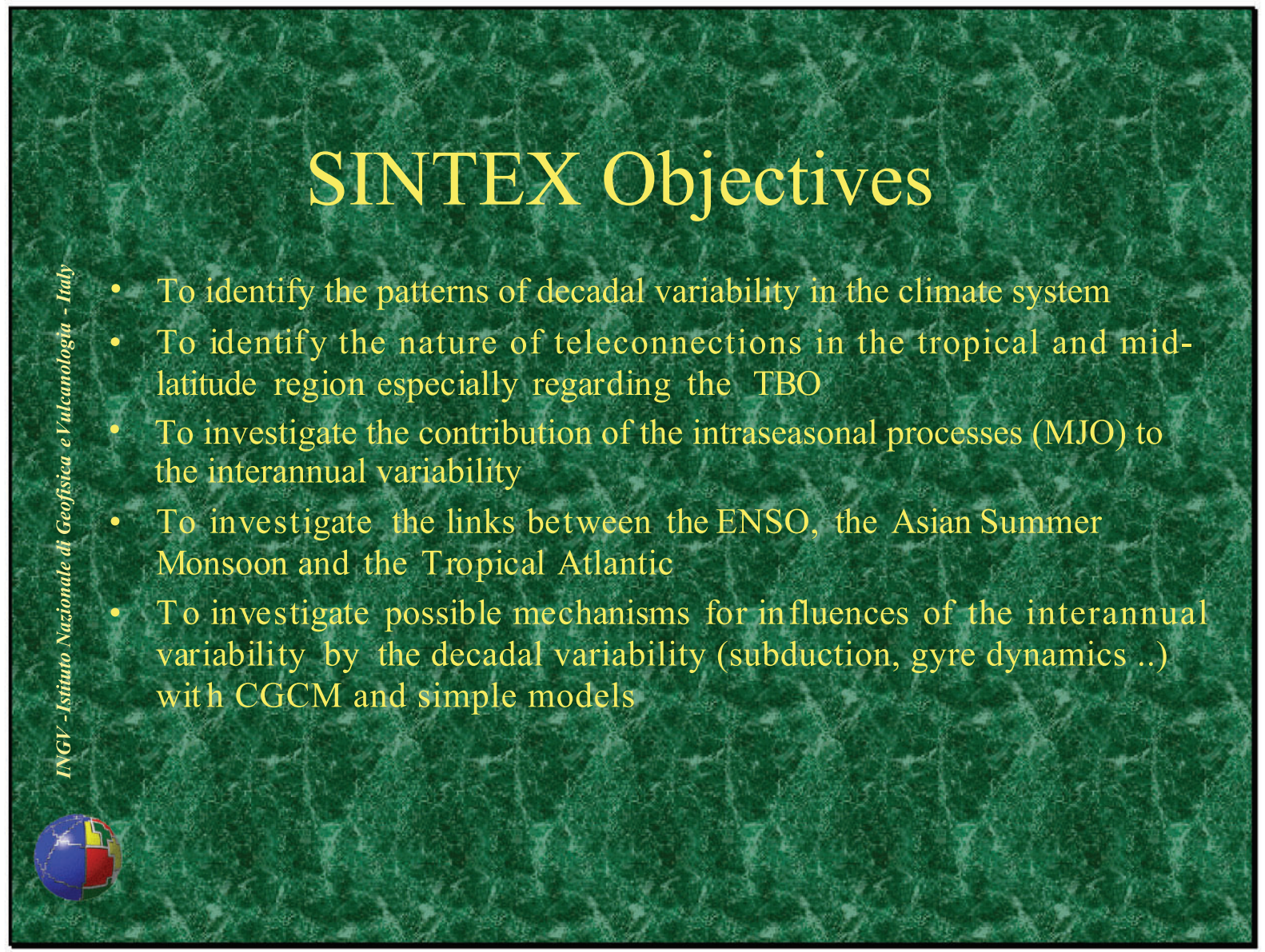

Fig. 3. The original slide of the SINTEX objectives (1997).

experimentations and observational studies. The plan evolved into a proposal and then a project: SINTEX. The first draft of the project was circulated among the proponents (fig. 2) in 1998 and after much discussion and rewriting was submitted for funding to the IV Framework Program after a few months. The proposal was successful and the activity started in the spring of 1998. The SINTEX principles were then set in stone (fig. 3).

During the preparatory work for SINTEX we reversed the method for the design of experiments that has been common in the field of climate numerical simulation for some time. Rather than adapt our scientific aims to the available computing resources, we considered the computers as experimental machines to be obtained for doing the kind of science we had in mind. We went through the exercise to assess what level of computation was necessary to perform our scientific plan and then aim for the minimum level of equipment necessary to reach that goal.

The main idea of the project was to make available a number of numerical experiments specifically designed to study the intraseasonal, interannual and decadal variability. After much discussion, a consensus was reached that a length of about 200 years was a desirable and sufficient length to allow a comfortable evaluation of the interactions. The project was then made possible thanks to the collaboration of the NEC Supercomputing company that 
entered the project as a full partner, providing the required computing capability for the duration of the project and software engineering and optimization expertise. In this way, we achieved a high concentration of computing resources per individual scientist at a very moderate cost.

Several models were then identified to implement the numerical experiments: the Hadley Center Unified Model, the ECHAM4-OPYC coupled model, and a new coupled model based on the untried combination of existing atmospheric and oceanic components. In the end, SINTEX used for the analysis a combination of specifically devised experiments, available experiments and observations.

The new coupled model, soon to be known as the SINTEX model, was based on the atmospheric ECHAM4 model and the ocean model ORCA, coupled with the OASIS coupler. The objective was to aim for a model that was capable of a realistic interannual variability without flux correction. The model was created by a concurrent multi-institutional effort distributed in space, as the groups responsible were divided between Italy and France, working on German and French models on a Japanese computer located and operated by our Danish partner in Denmark. Amazingly, the logistics worked very well, even if everything was done over the standard science communication network in Europe, but obviously our lives would have been a lot easier with faster communication lines.

There are a few general points that SINTEX has contributed to the general scientific community.

The first is that SINTEX has shown that an approach where a scientific goal is first identified and then a particular computing system designed to achive it is possible. After all, this is how von Neumann, Charney and friends designed their first experiments in Princeton in the late 1940s. The approach is not new to meteorology, but it was somewhat overlooked in recent years.

Second, SINTEX showed that resolution is also an issue in climate length experiments and significant changes in resolution, in this case horizontal resolution, can lead to different and new physics. We have just started to realize the impact of this point and as the analysis of high resolution experiments progresses it probably will become clearer. It is not a magic wand, problems do not disappear into tiny powdery clouds, but qualitatively different things happen.

Last, but not least, SINTEX showed the feasibility of having several research groups working together in a complex multinstitutional, multinational context. The trick here for the success of this model was to concentrate computing resources, and disperse human capital. Not everybody may agree, but our experience was that it is easier to network humans than computers.

This special issue does not contain all the SINTEX results. SINTEX generated more than 20 papers that have been published through the main scientific literature, but we felt that at the end of the project it was a useful idea to have an easy to reach reference that would give a flavor of SINTEX and at the same time point to the vast wealth of scientific results produced by SINTEX that could not be contained in a single special issue. The papers published here will give in some cases reference or baseline presentation of SINTEX activities. Hopefully, the issue will be useful to the reader as a guide to the whole of SINTEX. 
SINTEX has been an exciting and demanding professional and personal experience for several years, and it would not have been possible without the support and friendship of all the partners in the project. It is a pleasure to acknowledge here the contributions of Pascale Delecluse, Julia Slingo, Leif Larsen, Mark Rodwell, Rowan Sutton, Mojib Latif, Laurent Li, Mariá Ortiz, Claude Frankignoul, Toine Beckers, Silvio Gualdi, Eric Guilyardi, Susanna Corti, and all the students and post-graduates who contributed to SINTEX. Hopefully, most of them learnead something useful, someone even learned to ski.

A major part of the science business is to identify the right questions, good directions that let us learn a lot of new things. We can say that in SINTEX we have attacked several of the most relevant problems of climate dynamics today, made good advances in some cases, smaller steps in others, but we have solved none. This is science and this is how it progresses from one generation of scientists to the next. The individual results are in the SINTEX papers to be judged.

\section{ANTONIO NAVARRA \\ Coordinator of SINTEX}

Istituto Nazionale di Geofisica e Vulcanologia, Bologna, Italy 